\title{
Hoek-Brown rock mass: adjusting Geological Strength Index for directional strength
}

\author{
NRP Baczynski Prime Geotechnics Pty Ltd, Australia
}

\begin{abstract}
Rock mass shear strength is often directional due to sets of geological defects that co-align with failure paths. In the limit, the entire path may be defined by co-aligned defects and rock mass strength in that direction is equal to defect strength plus the strength of any intact rock bridges that may exist between such defects. Conceptual considerations (Baczynski 2018) and a large number of case studies (Baczynski 2019a, b) indicate strong linear relationships between relative occurrences (\%) of co-aligned defects and intact rock bridges and the adjustments required in the Geological Strength Index (GSI) input to Hoek-Brown equations to quantify the directional shear strength. GSI adjustment is a two-step process. The general rock mass GSI is first negatively adjusted for relative portion (\%) of failure path length that is defined by geological defects co-aligned with the path. GSI is then positively adjusted for relative portion (\%) of failure path length defined by intact rock bridges between the co-aligned defects. GSI (directional) is computed as GSI (general rock mass) minus GSI (defect adjustment) plus GSI (bridge adjustment). For general design, GSI (defect adjustment) is $0.4 \times$ (occurrence (\%) of co-aligned defects) and GSI (bridge adjustment) is $1.2 \times$ (occurrence (\%) of intact rock bridges along the failure path). Correlation coefficients for the proposed adjustments are typically 0.75 to 0.95. GSI adjustment factors may be further refined by $m_{i}$ and rock type. The geotechnical data that needs to be collected to enable GSI adjustment is discussed. Indicative step-path case study results used to develop the recommended GSI adjustments are shown. Example data for probabilities (\%) of occurrence for defects and intact rock bridges and their respective lengths are tabulated. Use of the Rosenblueth method to develop statistical models is explained.
\end{abstract}

Keywords: Hoek-Brown rock mass, directional strength, data collection, case studies, GSI adjustment

\section{Introduction}

GSI adjustment for Hoek-Brown directional strength model requires that relative occurrences (\%) of co-aligned defects and intact rock bridges are somehow determined. Different co-aligned defect sets are likely associated with each failure mode (i.e. as identified by kinematic stability analyses); hence, relative occurrences may need to be derived for several sets in the rock mass. GSI input to Hoek-Brown calculations is adjusted on the basis of the following equation (Baczynski 2019a, b):

GSI $($ directional $)=$ GSI (general rock mass $)-[0.4 \times(\%$ co-aligned defects $)]+[1.2 \times(\%$ intact rock bridges $)]$

Derivation of this equation, geotechnical data collection and its interpretation, and GSI adjustment steps are described. Field examples of relative occurrences (\%) for co-aligned defects and intact rock bridges are tabulated. Rock mass parameters are rarely constant but statistically variable. Use of the Rosenblueth method for the statistical to assess material strength variability and stability risks is explained.

\section{Basic strength considerations}

Viewed simplistically, a rock mass comprises two components: intact rock and geological defects. The following strength conditions exist.

- Geological defects are less strong than intact rock and rock mass. The only exception is where defects have strong infill (e.g. quartz) and intact rock is extremely weathered/decomposed to residual soils. 
- Isotropic intact rock compressive strength is scale-dependent: strength decreases with increasing test block size, but this decrease appears to plateau out at about one cubic metre size.

- Layered intact rock is often anisotropic and has directional compressive strength; this being least at about $30-40^{\circ}$ due to shear strength of the anisotropy (as seen in curves of strength anisotropy (e.g. Brady \& Brown 2004, p. 118) and highest at $90^{\circ}$ to it.

- Isotropic rock mass strength decreases with increasing intensity of geological defects or decrease in GSI as may be computed by considering the rock quality designation (RQD) index.

- Rock mass strength may be significantly impacted by orientation and effective continuity (i.e. not just the intensity) of geological defects. If a specific set of geological defects is significantly co-aligned with failure path direction then the rock mass strength is weaker in that direction. The resulting strength is deemed as being anisotropic or directional.

Effective continuity describes situations where while individual defects have limited length due to their spatial distribution and interconnection by cut-off and step-up on other defects, failure mostly occurs by shear sliding along co-aligned defects and not by shearing across the structural fabric in the rock mass.

The logical shear strength relationship is (as shown in Figure 1):

Intact rock strength > rock mass strength > step-path strength > defects strength

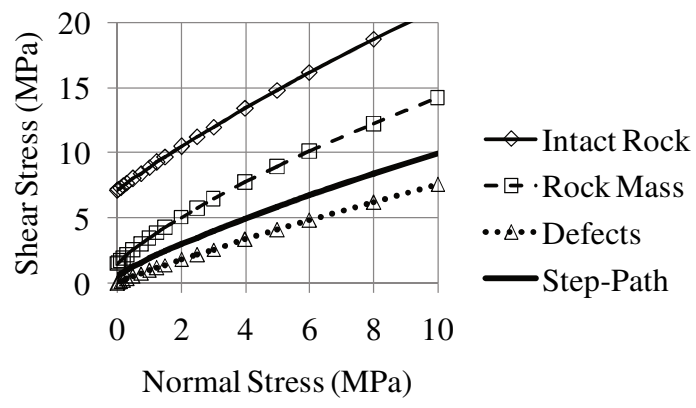

Figure 1 Conceptual strength relationships

Because step-path strength is generally less than the Hoek-Brown strength, limit equilibrium stability analyses (LEA) with step-path inputs tend to yield lower Factors of Safety (FS). FS decrease for slopes may be as high as 0.15 to 0.3 . This decrease is of similar magnitude to the FS difference often noted between LEA and jointed UDEC, FLAC and other numerical models (Baczynski et al. 2011; Bar \& Baczynski 2019).

\section{$3 \quad$ Kinematic stability}

Kinematic stability analyses identify potential failure modes (e.g. planar, tetrahedral wedge, active-passive wedge, circular, and toppling). Specific defect sets are associated with each mode. A directional strength model is required for each defect set impacting stability.

\section{$4 \quad$ Hoek-Brown directional strength}

The Hoek-Brown Method (HBM) does assess directional strength. This is achieved by adjusting the Geological Strength Index (GSI). Two factors are considered:

- Vertical axis: rock mass condition (mostly based on intensity of fracturing).

- Horizontal axis: geological defect condition (essentially related to defect strength).

Several versions of GSI charts have been published over last 30 years. However, most directional strength charts are significantly qualitative, not quantitative. The GSI chart presented by Truzman (2007) is perceived as the most useful; but, intensity on its vertical rock mass condition axis should ideally be renamed as relative occurrence (\%) of co-aligned defects to make this chart better suited to quantifying directional strength. 
No GSI chart considers the positive strength impact of intact rock bridges between the failure path co-aligned defects because of the original assumption of defect continuity. This consideration is important and should be considered. The strength will differ between rock mass situations where, for exactly the same effective co-aligned defect continuity, defects are mostly cut off by other defect sets (i.e. few intact rock bridges exist) and where they are mostly not cut off (i.e. considerable number of intact rock bridges exist).

Computer simulation (Baczynski 2018) and 230 step-path case studies (Baczynski 2019a, b) provide the basis for the author's GSI adjustment input to Hoek-Brown directional strength.

\section{$5 \quad$ Step-path directional strength}

The basic step-path shear strength concept is shown in Figure 2. Three cell strength types are considered. These are intact rock, geological defects (i.e. those co-aligned with failure path direction) and rock mass (i.e. where defects are not co-aligned with failure path direction). Strength is calculated by multiplying each of the three cell-type strengths by their relative occurrence along the failure path.

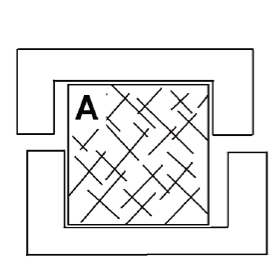

General rock mass

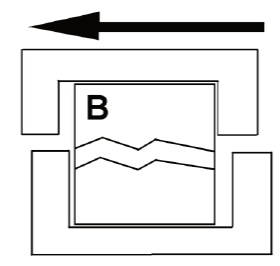

Co-aligned defect

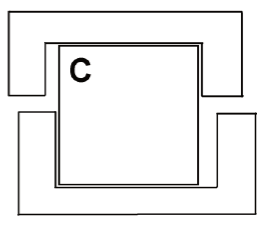

Intact Rock Bridge

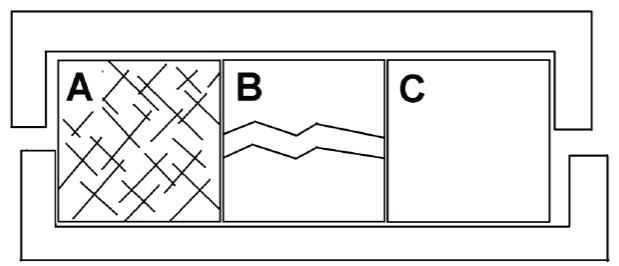

Failure path with one of each strength component

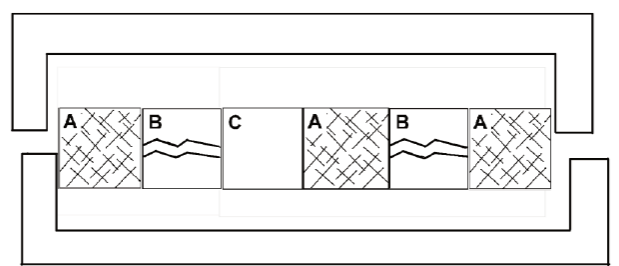

Failure path with statistical assemblage of strength components

Figure 2 Conceptual step-path strength model

If the failure path is $100 \%$ defined by co-aligned defects, then the step-path strength is defect strength. If the failure path is $100 \%$ intact rock, then the step-path strength is intact rock strength (suitably adjusted for scale effects). If the failure path is $100 \%$ defined by the rock mass, then the step-path strength is the Hoek-Brown strength. Conceptual examples of co-aligned defect step-path strength are shown in Figure 3 . In the northwest and northeast quadrants are examples of planar failure modes due to perfectly and echelon co-aligned defects and bridges between them. The southwest quadrant shows a more typical step-path; the failure path trends along one set and steps up on another steeper set. The southeast quadrant shows partial step-path strength (i.e. failure back-scarp is defined by a fault and Hoek-Brown strength along centre of failure path). Figure 4 shows the structural pattern, most likely failure path, and examples of structural components and strength components. Similar types of models and failure mode scenarios need to be developed for all rock slopes before shear strength parameters are finalised and stability analyses done. 


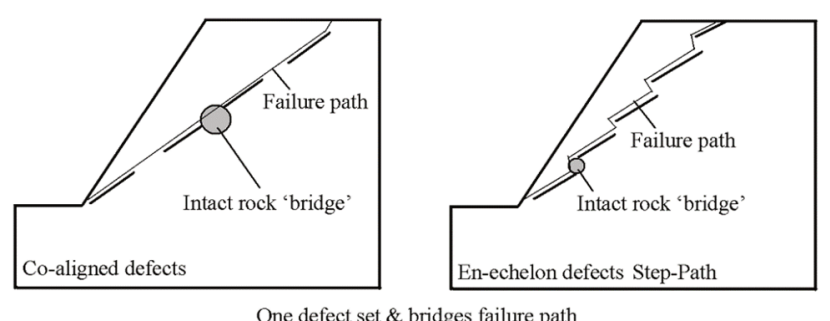

One defect set \& bridges failure path

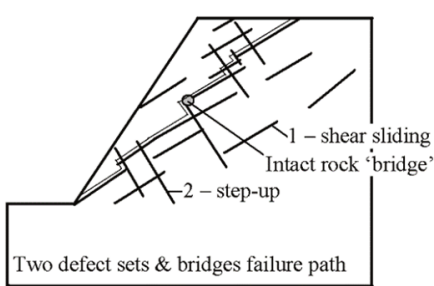

Step-Path

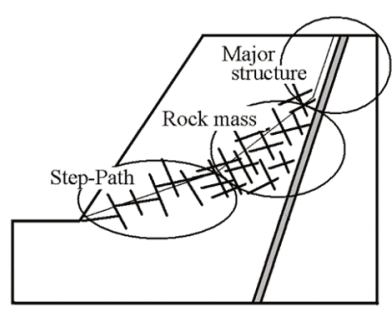

Composite failure path

Figure 3 Examples of various types of step-path (directional) strength in rock masses

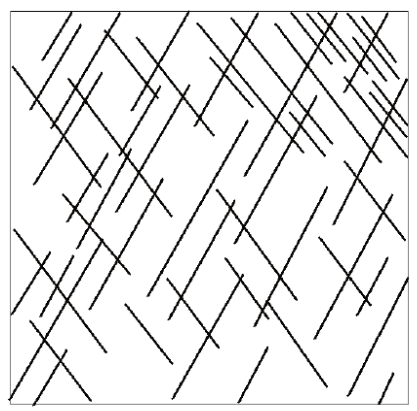

Rock mass defect pattern

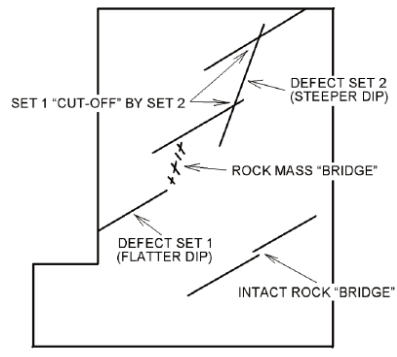

Structural components

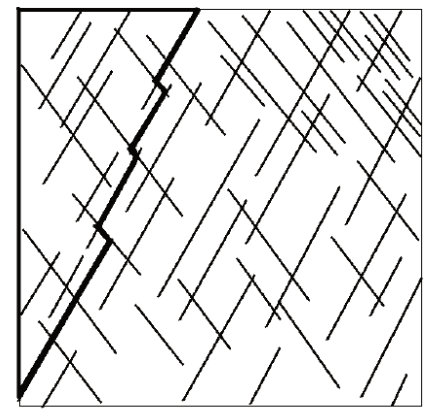

$100 \%$ defect-controlled failure path

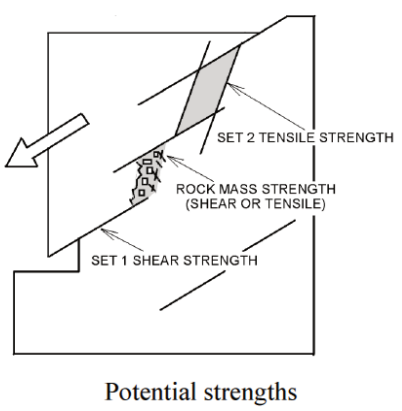

Figure 4 Conceptual example: structural pattern, 100\% defect-controlled failure path, rock mass strength components and potential failure mode/strength for each component

\section{GSI adjustment quantified by step-path case studies}

Baczynski (2019a, b) proposed the following general GSI adjustment equation:

GSI $($ design $)=$ GSI (general rock mass $)-[0.4 \times(\%$ co-aligned defects $)]+[1.2 \times(\%$ intact rock bridges $)]$

Figure 5 shows examples of some of the cross-correlations achieved between relative occurrences (\%) of co-aligned defects and intact rock bridges and GSI adjustments that were required to derive near-same step-path method and Hoek-Brown method shear strength models.

Based on review of 230 case studies, Table 1 summarises the GSI adjustment factor for different $m_{i}$ groups $(<11,11-19,>19)$ and for several rock types. 

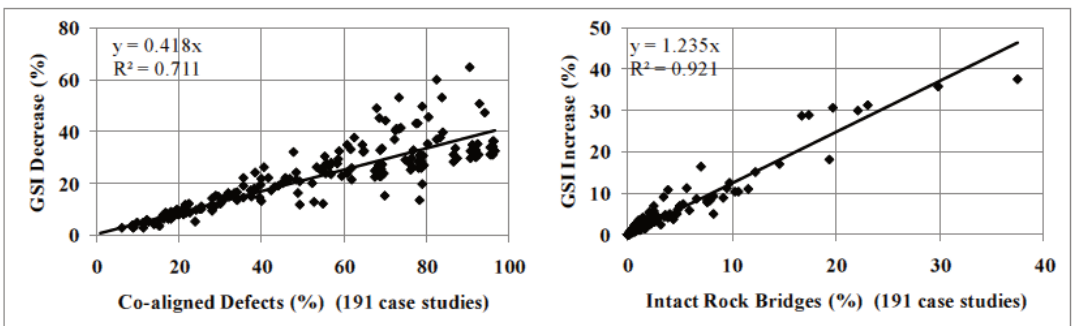

(A) All case studies combined
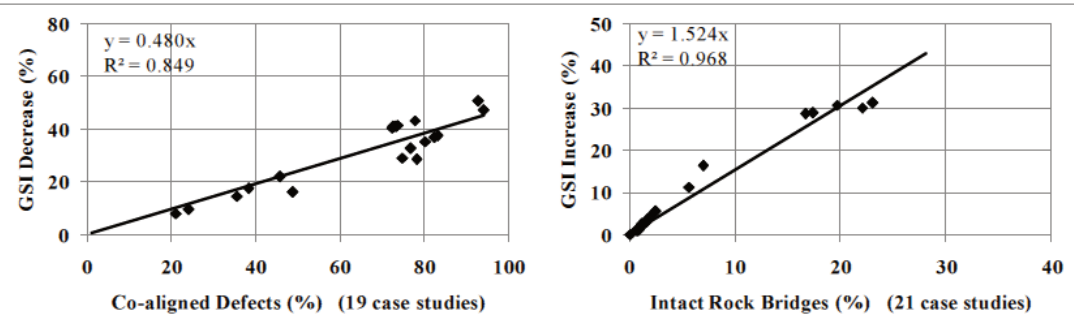

(B) Partitioned by $\mathrm{m}_{\mathrm{i}}<11$
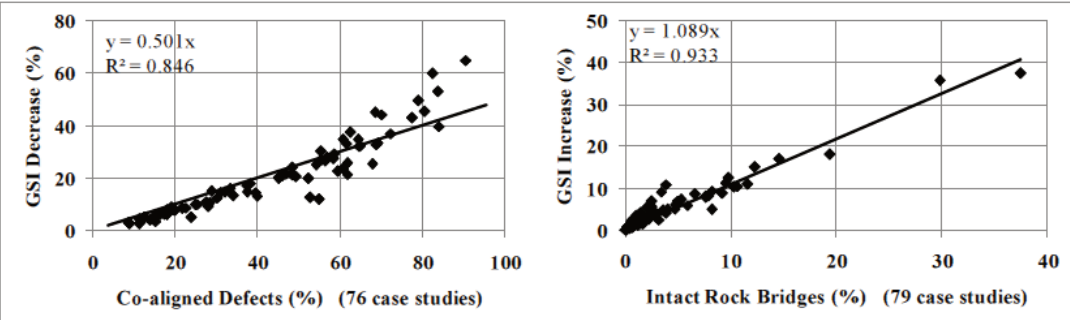

(C) Partitioned by $\mathrm{m}_{\mathrm{i}} 11-19$
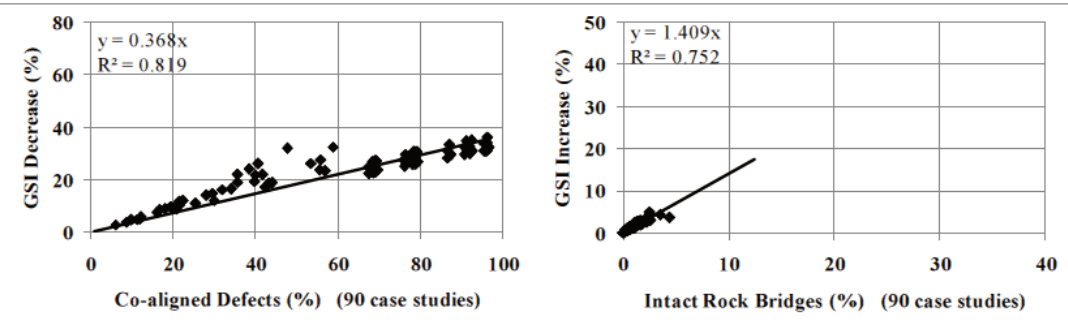

(D) Partitioned by $\mathrm{m}_{\mathrm{i}}>19$

Figure 5 Example: occurrence (\%) of co-aligned defects and intact rock bridges versus GSI adjustment to achieve near-same step-path and Hoek-Brown shear strength models for rock masses; all case studies combined and cases partitioned on basis of $m_{i}$ (i.e. $<11,11-19$ and $>19$ ) (Baczynski 2019a)

Table 1 Suggested GSI adjustment factors (rock mass GSI $\leq 75$ ) (Baczynski 2019a)

\begin{tabular}{lll}
\hline Rock type & \multicolumn{2}{l}{ GSI adjustment factor (AF) per 1\% occurrence } \\
\cline { 2 - 3 } & Co-aligned defects & Intact rock (IR) bridges \\
\hline General rock mass & 0.40 & 1.2 \\
Rock $\mathrm{m}_{\mathrm{i}} \leq 19$ & 0.48 & 1.2 \\
Rock $\mathrm{m}_{\mathrm{i}}>19$ & 0.35 & 1.2 \\
Sedimentary (all varieties) & 0.48 & 1.2 \\
Sandstone & 0.53 & 1.0 \\
Siltstone & 0.40 & 1.3 \\
Igneous (intrusive) & 0.37 & 1.5 \\
Metamorphic (schist only) & 0.53 & 2.0 \\
\hline
\end{tabular}




\section{$7 \quad$ Geotechnical investigations for directional strength}

Relative occurrences (\%) of co-aligned defects and intact rock bridges need to be estimated to suitably adjust GSI in the Hoek-Brown method. An experienced engineer might be able to make a reasonably good estimate of the relative occurrences by a walk-over inspection of slope faces, but this approach is only possible if potential slope failure modes (and associated geological defects) are already known, allowing the inspection to focus on specific defect sets. However, such prior knowledge is often not the situation. More commonly, there is no prior knowledge of likely slope failure modes, and enough field and laboratory data needs to be collected to develop statistical geological defect and rock mass attribute models. Kinematic stability analyses are only done after stereographic projection plots have been generated.

Data for step-path models and GSI adjustment involves conventional field and laboratory investigations. Whilst various structural mapping approaches exist (e.g. area mapping, window mapping, line traverse, photography), the author is a strong proponent of line traverse mapping of slope faces and underground openings. Line traverse data is directly comparable to drillcore data. Area mapping data is often swamped by shorter defects. Statistical models (e.g. for defect length) based on area mapping hence are not directly comparable to those derived by line traverse mapping. However, photo-mapped structural patterns may be line-traverse sampled to achieve the desired outcome; although photography provides little to no data on defect roughness, infill type, rock weathering grade and defect wall rock strength.

The following sections describe the input data required for a statistical evaluation.

\subsection{Intact rock}

The following data is required for each significant rock type: rock description (e.g. igneous, metamorphic, sedimentary, lithology, mineralogy, grain size, anisotropy layering), weathering, density, moisture content, unconfined compressive strength (UCS, including testing direction with respect to anisotropy), UCS tested at different directions to anisotropy (ideally), Is(50) point-load strength (both axial and diametral), Brazilian tensile strength, UCS - Is(50) strength ratio (axial and diametral), direct shear strength, base friction angle (for different weathering grades if relevant), triaxial compressive strength (TCS, ideally), HBM $\mathrm{m}_{\mathrm{i}}$ parameter (ideally determined on basis of project-specific TCS tests, otherwise estimated from already published data).

\subsection{Geological defects}

The following data is required: defect type (fault, joint, bedding, foliation, vein, etc.), location or distance along the line traverse/borehole, orientation, length, surface roughness (i.e. small-scale surface roughness measured over $0.1 \mathrm{~m}$ as per Barton's 1-10 scale, and large-scale undulations measured as wavelength and its amplitude and subsequently expressed as roughness angle (i), aperture (width), infill type (eventually grouped as none, weak, or strong), water seepage, wall rock strength and weathering grade, and termination type (i.e. whether the defect is cut off by other defects - yes/no). Either an intact rock (IR) or rock mass (RM) bridge exists between each defect that is not cut off by another defect (Figure 6). The following data is required for IR bridges: rock type, length, weathering grade, and UCS. The following data is required for RM bridges: rock type, length, intact rock UCS, weathering, fractures/m, RQD, rock mass rating (RMR), and GSI indices.

The peak and residual shear of each defect type should be laboratory tested and/or field estimated.

\subsection{Rock mass attributes}

General rock mass is characterised at regular slope face distance intervals (e.g. 10-20 m; depending on the total length of the line traverse; shorter intervals required if slope face is relatively short) in $3 \times 3 \mathrm{~m}$ size mapping windows, or systematically assessed over 3 to $5 \mathrm{~m}$ core logging intervals. The following data is required: rock type, description, anisotropic layering, intact rock UCS, weathering grade and classification rating (defects intensity/m, number of defect sets, RQD, RMR, and GSI). 


\subsection{Other considerations}

Drilling cannot determine defect lengths, large-scale defect surface undulations, defect cut-off by other defects and IR/RM bridge lengths. If only drilling is available, these attributes need to be estimated. Slope design solely based on core data is preliminary until confirmed by mapping of rock excavations.

\section{Data interpretation}

Data is validated and suspect data are corrected or deleted based on experience and observation. Stereographic projections are plotted. Defect sets are interpreted and potential failure modes identified. Statistical models are developed; efforts should concentrate on defect sets likely to impact stability. Figures $6 \mathrm{a}$ and $\mathrm{b}$ show how the probability of defect occurrence and cut-off are estimated. The borehole or line traverse data is divided into segments (e.g. 0-5 m, 5-10 m, 10-15 m, etc.). The proportion (\%) of segments that encounter specific defect sets is assessed. If a set occurs in $65 \%$ of the segments, then its relative occurrence is considered to be $65 \%$.
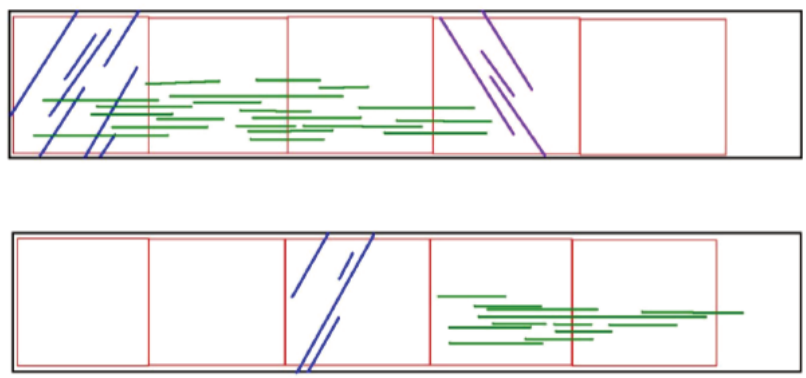

Probability of Occurrence:

Set $1=20 \% \square$ Set $2=10 \% \square$ Set $3=60 \%$

(A)

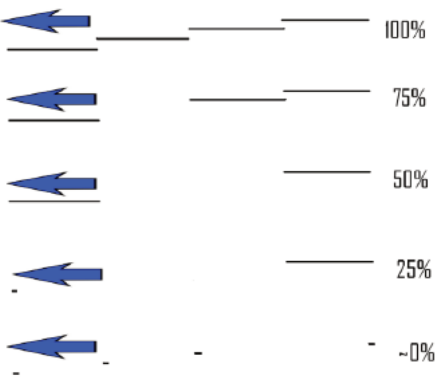

(B)

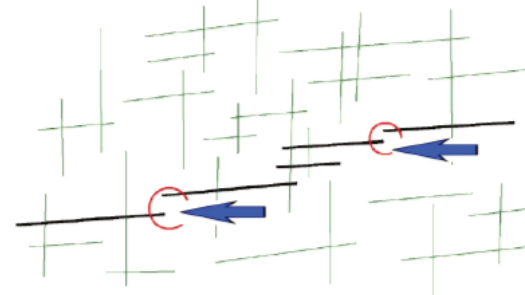

(D)

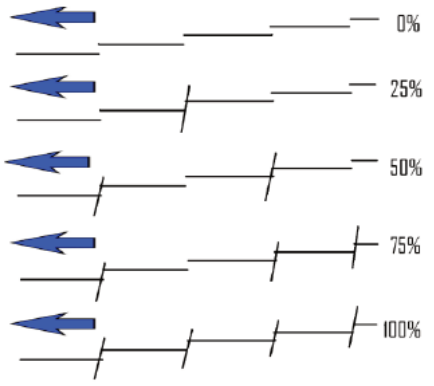

(C)

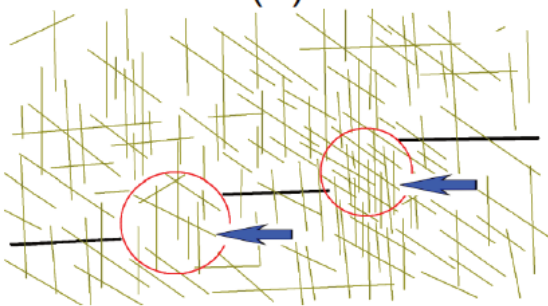

(E)

Figure 6 Concepts: (a, b) Probability of defect occurrence; (c) Defect cut-off by other defects; (d) Intact rock bridges; and (e) Rock mass bridges

Figure $6 \mathrm{c}$ shows defect cut-off by other defects. Figures $6 \mathrm{~d}$ and e show intact rock and rock mass bridges.

If sufficient field data exists (i.e. systematic line traverse mapping around the entire perimeter of an open pit), it may be statistically meaningful for the project area to be divided into several domains, with different probability of defect occurrence characterising each domain. 


\section{Calculation of failure path strength}

The following information is collated after data interpretation for each defect type (faults, bedding or joints) that belong to the specific co-aligned defect set orientation that is being considered:

- Probability of occurrence (\%) of the specific co-aligned defects (0-100\%) in the rock mass.

- Probability of occurrence (\%) of rock mass zones where this specific co-aligned defect set is absent (100\% less probability of the specific co-aligned defect occurrence).

- Relative percentages (\%) of this specific co-aligned defect set's members are partitioned by defect type (e.g. faults and joints or bedding shears and bedding planes) with each defect type further partitioned by infill type (strong, none, weak).

- This co-aligned defect set's length attributes for each defect type (mean \pm standard deviation).

- Probability (\%) that members of this co-aligned defect set are cut off by other defect sets (0-100\%) for each defect type.

- Attributes of bridges for this co-aligned set's members that are not cut off by other defect sets for each defect type (faults, bedding, joints).

- Probability of occurrence (\%) for bridges (100\% minus probability of cut-off) for each defect type (faults, bedding, joints).

- Relative percentage (\%) of each bridge type (intact rock, rock mass) for each defect type.

- Intact rock bridge length (mean \pm standard deviation) for each defect type (fault, joint, etc.).

- Rock mass bridge length (mean \pm standard deviation) for each defect type.

Table 2 shows case study examples of probability for occurrence (\%) along potential rock mass failure paths and mean length for co-aligned defects and intact rock bridges, respectively.

The next analysis step is to compute for each co-aligned defect set what proportion (\%) of slope failure path will comprise:

1. Rock mass zones without co-aligned defects.

2. Co-aligned defects (if applicable, further partitioned by type: faults, joints and by their infill types).

3. Intact rock bridges.

4. Rock mass bridges.

If the probability of defect cut-off (by other defects) is high, then the occurrence of bridges is conversely low. Standard deviation for length of defects and intact rock bridges is often $50 \%$ to $80 \%$ of the mean values indicated in Table 2.

The simplest models consider only mean inputs for each of the above four parameters. Conventional approaches (e.g. Hoek-Brown rock mass and Barton's joint equations) can be used to assess shear strength of each component.

If rock mass bridges and general rock mass zones are the same strength and all co-aligned defects are the same type (either all faults or all joints), bullet points 1 and 4 are summed; the general rock mass GSI is adjusted as per the equation presented in Section 6 and directional shear determined by Hoek-Brown equations. For example, if the general GSI was 65 for joint set J1 in the fourth row of Table 2, then the adjusted GSI would be calculated via $(65.0-[0.4 \times 60.7]+[1.2 \times 12.3])$. This simplifies to $(65.0-24.3+14.8)$ and equals about 55 . In this example both the negative and positive adjustments were significant. If just the negative adjustment for defects had been considered with existence of bridges ignored, then GSI would have been negatively overcorrected and rock mass strength underestimated. 
For structurally complex rock masses where strength of rock mass zones and rock mass bridges is different and/or where the co-aligned defect comprises several types (i.e. faults, strong joints, weak joints), then failure path strength is likely easiest derived by using Excel spreadsheets, as shown in Figure 7.

Table 2 Examples of occurrences and lengths: geological defects and intact rock bridges

\begin{tabular}{|c|c|c|c|c|c|c|c|c|}
\hline \multirow[t]{2}{*}{ Project } & \multirow{2}{*}{$\begin{array}{l}\text { Rock } \\
\text { type }\end{array}$} & \multirow{2}{*}{$\begin{array}{l}\text { Joint } \\
\text { set }\end{array}$} & \multicolumn{2}{|c|}{ Ave. orientation } & \multicolumn{2}{|l|}{ Defects } & \multicolumn{2}{|c|}{ Intact rock bridges } \\
\hline & & & $\begin{array}{l}\text { Dip } \\
\text { direction } \\
\left({ }^{\circ}\right)\end{array}$ & $\begin{array}{l}\text { Dip } \\
\text { angle } \\
\left({ }^{\circ}\right)\end{array}$ & $\begin{array}{l}\text { Occurrence } \\
\text { (\%) }\end{array}$ & $\begin{array}{l}\text { Mean } \\
\text { length } \\
\text { (m) }\end{array}$ & $\begin{array}{l}\text { Occurrence } \\
\text { (\%) }\end{array}$ & $\begin{array}{l}\text { Mean } \\
\text { length } \\
(\mathrm{m})\end{array}$ \\
\hline Mt Owen & Siltstone (SL) & $\mathrm{J} 1$ & 260 & 45 & 78.3 & 2.6 & 1.7 & 0.2 \\
\hline \multirow[t]{2}{*}{ NSW } & SS-SL & $J 2$ & 200 & 85 & 15.2 & 1.5 & 0.8 & 0.3 \\
\hline & Sandstone (SS) & $\mathrm{J} 3$ & 290 & 89 & 64.4 & 10.0 & 0.6 & 0.2 \\
\hline Ravensworth & Sandstone & J1 & 125 & 88 & 60.7 & 2.7 & 12.3 & 0.6 \\
\hline \multirow[t]{2}{*}{ NSW } & & $J 2$ & 219 & 80 & 55.4 & 2.3 & 14.6 & 0.6 \\
\hline & & $\mathrm{J} 3$ & 243 & 85 & 33.4 & 2.5 & 10.6 & 0.8 \\
\hline Millennium & Siltstone & $\mathrm{J} 1$ & 135 & 85 & 68.8 & 2.6 & 9.2 & 0.35 \\
\hline \multirow[t]{2}{*}{ Qld } & & $J 2$ & 217 & 87 & 48.4 & 2.1 & 11.6 & 0.5 \\
\hline & & $\mathrm{J} 3$ & 090 & 89 & 58.7 & 1.5 & 10.4 & 0.27 \\
\hline Ok Tedi & Monzodiorite & $J 2$ & 347 & 33 & 29.9 & 5.6 & 1.1 & 0.35 \\
\hline \multirow[t]{11}{*}{ PNG } & & $J 4$ & 100 & 45 & 42.4 & 6.3 & 1.6 & 0.32 \\
\hline & & $\mathrm{J7}$ & 325 & 80 & 56.9 & 8.0 & 2.1 & 0.37 \\
\hline & & J9B & 230 & 88 & 26.5 & 6.5 & 1.5 & 0.53 \\
\hline & Siltstone & $\mathrm{J} 1$ & 282 & 47 & 69.2 & 3.2 & 9.8 & 0.56 \\
\hline & & $J 2$ & 000 & 40 & 18.1 & 3.4 & 1.9 & 0.57 \\
\hline & & $\mathrm{J} 3$ & 040 & 35 & 19.1 & 4.8 & 1.9 & 0.49 \\
\hline & & J5 & 185 & 85 & 55.4 & 4.0 & 6.6 & 0.6 \\
\hline & & $J 7$ & 275 & 89 & 31.0 & 3.4 & 4.0 & 0.5 \\
\hline & Monzonite & $J 2$ & 352 & 60 & 19.5 & 10.5 & 0.5 & 0.6 \\
\hline & & $\mathrm{J} 7$ & 295 & 83 & 16.0 & 9.3 & 1.0 & 0.87 \\
\hline & & J9 & 055 & 80 & 32.0 & 10.4 & 1.0 & 0.74 \\
\hline
\end{tabular}

Note: NSW - New South Wales, Qld - Queensland, PNG - Papua New Guinea

Risk-based models, developed using the Rosenblueth method of statistical moments (Rosenblueth 1975), consider all combinations of -1 and +1 standard deviation values for inputs. Figure 8 shows an example of a Rosenblueth calculation table used to assess Hoek-Brown rock mass strength.

The number of Rosenblueth calculations is $2^{\mathrm{N}}$, where $\mathrm{N}$ is the number of input components being considered. For example, since there are six strength components in Figure 8 , then $2^{6}$ or 64 Rosenblueth calculations will need to be done to develop a statistical strength model (for a rock mass with the tabulated probabilities of occurrence for the respective components). 
Alternatively, the aforementioned strength model may be developed via Monte Carlo simulation using software such as STEPSIM4 (as described in Baczynski 2000).

\begin{tabular}{|c|c|c|c|c|c|c|c|c|}
\hline 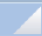 & A & B & C & D & $E$ & $\mathrm{~F}$ & G & H \\
\hline 1 & \multicolumn{8}{|c|}{ Example of more-detailed Simplified Step-Path Method (SSPM) EXCEL Spreadsheet } \\
\hline 2 & Overall Occurrences $(\%)$ & \multicolumn{2}{|c|}{5} & 35 & \multicolumn{3}{|c|}{60} & \\
\hline 3 & Occurrence by Type (\%) & 2 & 3 & 35 & 10 & 30 & 20 & \\
\hline 4 & & \multicolumn{2}{|c|}{ bridges } & Rock mass & \multicolumn{3}{|c|}{ Co-aligned defects } & \\
\hline 5 & & Intact & Rock & Zones without & Weak & Weak & Strong & \\
\hline 6 & Normal & Rock & Mass & co-aligned & Faults & Joints & Joints & \\
\hline 7 & Stress & & & defects & & & & Step-Path \\
\hline 8 & (MPa) & UCS 50 & UCS 30 & UCS 50 & UCS 1 & UCS 5 & UCS 50 & Model \\
\hline 9 & & $\operatorname{mi} 15$ & $\operatorname{mi} 15$ & $\operatorname{mi} 15$ & JRC 1 & JRC 2 & JRC 10 & $(\mathrm{SPM})$ \\
\hline 10 & & GSI 100 & GSI 50 & GSI 50 & base fri 22 & base fri 26 & base fri 30 & \\
\hline 11 & & \multicolumn{7}{|c|}{ Shear Stress (MPa) } \\
\hline 12 & 0.00 & 7.711 & 0.176 & 0.292 & 0.000 & 0.001 & 0.000 & 0.262 \\
\hline 13 & 0.10 & 7.880 & 0.415 & 0.545 & 0.042 & 0.056 & 0.154 & 0.413 \\
\hline 14 & 0.30 & 8.212 & 0.783 & 0.953 & 0.123 & 0.163 & 0.387 & 0.660 \\
\hline 15 & 0.50 & 8.540 & 1.093 & 1.308 & 0.202 & 0.266 & 0.596 & 0.881 \\
\hline 16 & 1.00 & 9.339 & 1.751 & 2.059 & 0.398 & 0.518 & 1.072 & 1.370 \\
\hline 17 & 2.00 & 10.860 & 2.830 & 3.310 & 0.784 & 1.010 & 1.930 & 2.228 \\
\hline 18 & 4.00 & 13.690 & 4.565 & 5.352 & 1.543 & 1.968 & 3.473 & 3.723 \\
\hline 19 & 6.00 & 16.280 & 6.023 & 7.080 & 2.294 & 2.906 & 4.895 & 5.064 \\
\hline 20 & 10.00 & 21.020 & 8.490 & 10.040 & 3.778 & 4.748 & 7.533 & 7.498 \\
\hline
\end{tabular}

Figure 7 Example of Excel spreadsheet use to develop step-path strength models (stated occurrence is the cumulative length of the specified component along the failure path)

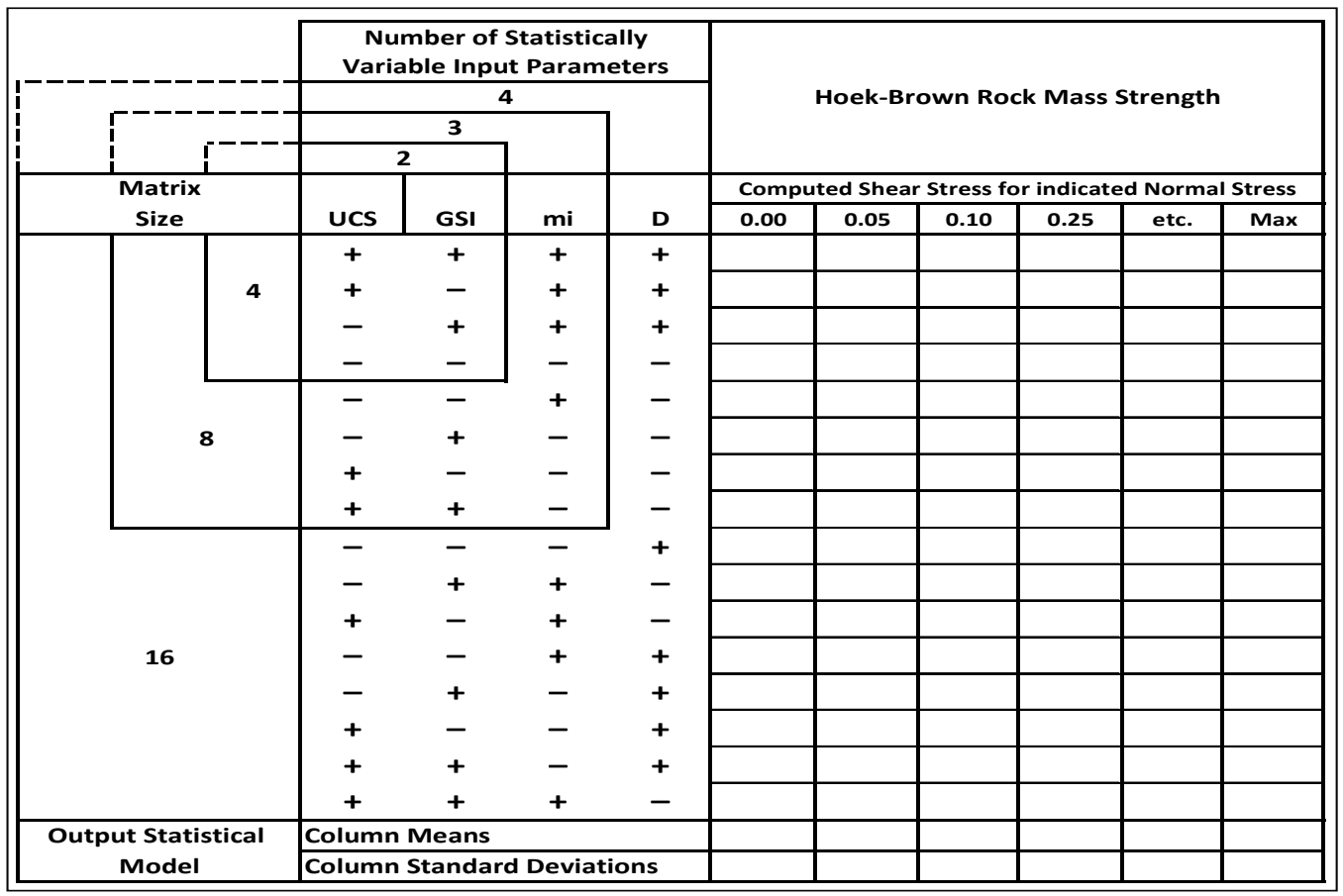

Figure 8 Rosenblueth method: example chart for combinations of -1 and +1 standard deviations

\section{Conclusions}

Rock masses are rarely homogeneous and isotropic. Failure modes are not always circular or quasi-circular.

All potential failure modes need to be identified to be able to estimate the most relevant rock mass strength parameters and to pursue the most appropriate limiting equilibrium stability assessment. Planar, wedge and toppling failures may be possible, depending on defect orientations. Different defect sets will likely impact different segments of potential failure paths. For example, near-vertical defects will co-align and impact 
back-scarp strength and near-horizontal defects (e.g. bedding planes) impact toe regions of circular paths through rock mass slopes.

Based on kinematic stability analyses, slopes should be partitioned into structural/kinematic domains. Each domain is associated with a specific defect set co-aligned with failure path direction. Domain boundaries are not fixed but likely differ for each failure mode being considered. Intact rock, rock mass and defect attributes are statistically variable. Accordingly, rock mass strength is likewise variable. Assessment of defect-controlled rock mass strength is fairly straightforward if defects have $100 \%$ continuity through the mass. This task is more involved if individual defects are relatively short with respect to scale of the rock excavation being considered. In the latter, directional strength is related to the percentage of the failure path that coincides with the co-aligned and cut off interconnected defects. Directional strength is likely best assessed by using step-path methods. However, these methods have not been widely adopted. Hoek-Brown does assess directional strength by adjusting the GSI. Other inputs to Hoek-Brown (i.e. UCS and $m_{i}$ ) are also significantly impacted by anisotropy but, as per recommended procedures, these inputs should be derived by testing samples in the direction normal to anisotropy.

Most GSI charts are significantly qualitative. Quantitative GSI charts relate rock mass condition to RQD or akin volumetric joint count; but RQD depends on intensity of geological fracturing, irrespective of defect orientations, and thus cannot be used to quantify directional strength. For directional strength, GSI is quantified by considering the relative occurrence (\%) of co-aligned defects and intact rock bridges. As per Baczynski (2019a, b), GSI adjustments are computed via the following equation:

GSI $($ directional $)=$ GSI (general rock mass $)-[0.4 \times(\%$ co-aligned defects $)]+[1.2 \times(\%$ intact rock bridges $)]$

The above GSI adjustment needs to be computed and applied in each anisotropy direction where a specific defect co-aligns with all or part of the failure path direction through the rock mass.

Relative occurrences of co-aligned defects and intact rock bridges are determined by conventional field and laboratory investigations. Required data, and its collection, analysis and interpretation are briefly described. Case study examples of data and GSI adjustment calculations are presented. Statistical models may be developed by using the Rosenblueth method. Statistical variability is scale-dependent, and may be adjusted as per sampling theory considerations.

Where checked, limit equilibrium stability analyses with strength inputs based on step-path and Hoek-Brown with above described GSI adjustments yield FS that are similar to those computed by jointed numerical models.

\section{References}

Baczynski, NRP 2000, 'STEPSIM4 Step-Path method for slope risks', Proceedings of GeoEng 2000: An International Conference on Geotechnical \& Geological Engineering, vol. 2, International Society for Rock Mechanics, Lisbon, p. 86.

Baczynski, NRP, deBruyn, IA, Mylvaganam, J \& Walker, DJH 2011, 'High rock slope cutback geotechnics: a case study at Ok Tedi Mine', in E Eberhardt \& D Stead (eds), Proceedings of Slope Stability 2011: International Symposium on Rock Slope Stability in Open Pit Mining and Civil Engineering, Canadian Rock Mechanics Association, $13 \mathrm{p}$.

Baczynski, NRP 2018, 'Step path adjusted Hoek Brown GSI chart', Proceedings of the 10th Asian Rock Mechanics Symposium, Society for Rock Mechanics and Engineering Geology, Singapore, paper 0590, $12 \mathrm{p}$.

Baczynski, NRP 2019a, 'GSI adjustment for directional Hoek-Brown strength quantified by case studies', Proceedings of the 14th ISRM Congress, International Society for Rock Mechanics, Lisbon, $8 \mathrm{p}$.

Baczynski, NRP 2019b, 'GSI adjustment for directional Hoek-Brown strength calibrated by step-path case studies', Australian Geomechanics, vol. 54, no. 3, pp. 51-78.

Bar, N \& Baczynski, NRP 2019, 'Slope failure risk by engineering geological logic and the simplified step-path method', Proceedings of the 13th Australia New Zealand Conference on Geomechanics, Australian Geomechanics Society, Perth, 5 p.

Brady, BHG \& Brown, ET 2004, Rock Mechanics for Underground Mining, 3rd edn, Kluwer Academic Publishers, Berlin.

Rosenblueth, E 1975, 'Point estimates of probability moments', Proceedings of the National Academy of Sciences, vol. 72, no. 10, pp. 3812-3814.

Truzman, M 2007, 'Statistical summary of rock mass characterization for tunnels of the Caracas-Tuy Medio railroad project', Proceedings of the XXIII Pan-American Conference on Soil Mechanics and Geotechnical Engineering, vol. 2, Sociedad Venezolana de Geotecnia, Chacao, pp. 877-882. 
